\title{
A note on the off-diagonal Muckenhoupt-Wheeden conjecture
}

\author{
David Cruz-Uribe, SFO \\ Dept. of Mathematics, Trinity College, \\ Hartford, CT 06106-3100, USA \\ E-mail: david.cruzuribe@trincoll.edu \\ José María Martell \\ Instituto de Ciencias Matemáticas CSIC-UAM-UC3M-UCM \\ Consejo Superior de Investigaciones Cientificas \\ C/ Nicolás Cabrera, 13-15, E-28049 Madrid, Spain, \\ E-mail: chema.martell@icmat.es \\ Carlos Pérez \\ Departamento de Análisis Matemático, \\ Facultad de Matemáticas, Universidad de Sevilla, \\ 41012 Sevilla, Spain \\ E-mail: carlosperez@us.es
}

We obtain the off-diagonal Muckenhoupt-Wheeden conjecture for CalderónZygmund operators. Namely, given $1<p<q<\infty$ and a pair of weights $(u, v)$, if the Hardy-Littlewood maximal function satisfies the following two weight inequalities:

$$
M: L^{p}(v) \rightarrow L^{q}(u) \quad \text { and } \quad M: L^{q^{\prime}}\left(u^{1-q^{\prime}}\right) \rightarrow L^{p^{\prime}}\left(v^{1-p^{\prime}}\right),
$$

then any Calderón-Zygmund operator $T$ and its associated truncated maximal operator $T_{\star}$ are bounded from $L^{p}(v)$ to $L^{q}(u)$. Additionally, assuming only the second estimate for $M$ then $T$ and $T_{\star}$ map continuously $L^{p}(v)$ into $L^{q, \infty}(u)$. We also consider the case of generalized Haar shift operators and show that their off-diagonal two weight estimates are governed by the corresponding estimates for the dyadic Hardy-Littlewood maximal function.

Keywords: Haar shift operators; Calderón-Zygmund operators; two-weight inequalities; testing conditions.

The first author was supported by a grant from the Faculty Research Committee and the Stewart-Dorwart Faculty Development Fund at Trinity College; the first and third authors are supported by grant MTM2009-08934 from the Spanish Ministry of Science and Innovation; the second author is supported by grant Grant MTM2010-16518 from the same institution

2000 Mathematics Subject Classification: 42B20, 42B25. 


\section{Introduction and Main results}

In the 1970s, Muckenhoupt and Wheeden conjectured that given $p$, $1<p<\infty$, a sufficient condition for the Hilbert transform to satisfy the two weight norm inequality

$$
H: L^{p}(v) \rightarrow L^{p}(u)
$$

is that the Hardy-Littlewood maximal operator satisfy the pair of norm inequalities

$$
\begin{aligned}
M: L^{p}(v) & \rightarrow L^{p}(u), \\
M: L^{p^{\prime}}\left(u^{1-p^{\prime}}\right) & \rightarrow L^{p^{\prime}}\left(v^{1-p^{\prime}}\right) .
\end{aligned}
$$

Moreover, they conjectured that the Hilbert transform satisfies the weaktype inequality

$$
H: L^{p}(v) \rightarrow L^{p, \infty}(u)
$$

provided that the maximal operator satisfies the second "dual" inequality. Both of these conjectures readily extend to all Calderón-Zygmund operators (see the definition below). Very recently, both conjectures were disproved: the strong-type inequality by Reguera and Scurry ${ }^{1}$ and the weak-type inequality by the first author, Reznikov and Volberg ${ }^{2}$.

Remark 1.1. A special case of these conjectures, involving the $A_{p}$ bump conditions, has been considered by several authors: $\operatorname{see}^{2-7}$.

In this note we prove the somewhat surprising fact that the Muckenhoupt-Wheeden conjectures are true for off-diagonal inequalities. Our main result is Theorem 1.1 below. We also prove an analogous result for the Haar shift operators (the so-called dyadic Calderón-Zygmund operators) with the Hardy-Littlewood maximal operator replaced by the dyadic maximal operator: see Theorem 1.2 below.

To state our results we first give some preliminary definitions. By weights we will always mean non-negative, measurable functions. Given a pair of weights $(u, v)$, hereafter we will assume that $u>0$ on a set of positive measure and $u<\infty$ a.e., and $v>0$ a.e. and $v<\infty$ on a set of positive measure. We will also use the standard notation $0 \cdot \infty=0$. 


\section{Calderón-Zygmund operators}

A Calderón-Zygmund operator $T$ is a linear operator that is bounded on $L^{2}\left(\mathbb{R}^{n}\right)$ and

$$
T f(x)=\int_{\mathbb{R}^{n}} K(x, y) f(y) d y, \quad f \in L_{c}^{\infty}\left(\mathbb{R}^{n}\right), \quad x \notin \operatorname{supp} f,
$$

where the kernel $K$ satisfies the size and smoothness estimates

$$
|K(x, y)| \leq \frac{C}{|x-y|^{n}}, \quad x \neq y
$$

and

$$
\left|K(x, y)-K\left(x^{\prime}, y\right)\right|+\left|K(y, x)-K\left(y, x^{\prime}\right)\right| \leq C \frac{\left|x-x^{\prime}\right|^{\delta}}{|x-y|^{n+\delta}},
$$

for all $|x-y|>2\left|x-x^{\prime}\right|$.

Associated with $T$ is the truncated maximal operator

$$
T_{\star} f(x)=\sup _{0<\epsilon<\epsilon^{\prime}<\infty}\left|\int_{\epsilon<|x-y|<\epsilon^{\prime}} K(x, y) f(y) d y\right| .
$$

Let $M$ denote the Hardy-Littlewood maximal operator, that is,

$$
M f(x)=\sup _{Q \ni x} f_{Q}|f(y)| d y=\sup _{Q \ni x} \frac{1}{|Q|} \int_{Q}|f(y)| d y .
$$

where the supremum is taken over all cubes in $\mathbb{R}^{n}$ with sides parallel to the coordinate axes.

Theorem 1.1. Given a Calderón-Zygmund operator T, let $1<p<q<\infty$ and let $(u, v)$ be a pair of weights. If the maximal operator satisfies

$$
M: L^{p}(v) \rightarrow L^{q}(u) \quad \text { and } \quad M: L^{q^{\prime}}\left(u^{1-q^{\prime}}\right) \rightarrow L^{p^{\prime}}\left(v^{1-p^{\prime}}\right)
$$

then

$$
\|T f\|_{L^{q}(u)} \leq C\|f\|_{L^{p}(v)} \quad \text { and } \quad\left\|T_{\star} f\right\|_{L^{q}(u)} \leq C\|f\|_{L^{p}(v)} .
$$

Analogously, if the maximal operator satisfies

$$
M: L^{q^{\prime}}\left(u^{1-q^{\prime}}\right) \rightarrow L^{p^{\prime}}\left(v^{1-p^{\prime}}\right),
$$

then

$$
\|T f\|_{L^{q, \infty}(u)} \leq C\|f\|_{L^{p}(v)} \quad \text { and } \quad\left\|T_{\star} f\right\|_{L^{q, \infty}(u)} \leq C\|f\|_{L^{p}(v)} .
$$


If the pairs of weights $(u, v)$ satisfy any of the conditions in (1), then the weights $u$ and $v^{1-p^{\prime}}$ are locally integrable. This is a consequence of a characterization of the two weight norm inequalities for the maximal operator due to Sawyer ${ }^{8}$. He proved that the $L^{p}-L^{q}$ inequality holds if and only if for every cube $Q$,

$$
\left(\int_{Q} M\left(v^{1-p^{\prime}} \chi_{Q}\right)(x)^{q} u(x) d x\right)^{1 / q} \leq C\left(\int_{Q} v(x)^{1-p^{\prime}} d x\right)^{1 / p}<\infty,
$$

and the $L^{q^{\prime}}-L^{p^{\prime}}$ inequality holds if and only if

$$
\left(\int_{Q} M\left(u \chi_{Q}\right)(x)^{p^{\prime}} v(x)^{1-p^{\prime}} d x\right)^{1 / p^{\prime}} \leq C\left(\int_{Q} u(x) d x\right)^{1 / q^{\prime}}<\infty .
$$

It is straightforward to construct pairs of weights that satisfy these conditions. For instance, in $\mathbb{R}$ both of these conditions follow easily for every $1<p \leq q<\infty$ and the pair of weights $(u, v)$ with $u=\chi_{[0,1]}$ and $v^{-1}=\chi_{[2,3]}$ (i.e., $v=1$ in $[2,3]$ and $v=\infty$ elsewhere). Indeed, we only need to check Sawyer's inequalities for cubes $\mathrm{Q}$ that intersect both $[0,1]$ and $[2,3]$, in which case we have $M\left(\chi_{[2,3] \cap Q}\right)(x) \leq|[2,3] \cap Q|$ for every $x \in[0,1] \cap Q$, and $M\left(\chi_{[0,1] \cap Q}\right)(x) \leq|[0,1] \cap Q|$ for every $x \in[2,3] \cap Q$. These readily imply the desired estimates.

\section{Dyadic Calderón-Zygmund operators}

A generalized dyadic grid $\mathscr{D}$ in $\mathbb{R}^{n}$ is a set of generalized dyadic cubes with the following properties: if $Q \in \mathscr{D}$ then $\ell(Q)=2^{k}, k \in \mathbb{Z}$; if $Q, R \in \mathscr{D}$ and $Q \cap R \neq \varnothing$ then $Q \subset R$ or $R \subset Q$; the cubes in $\mathscr{D}$ with $\ell(Q)=2^{-k}$ form a disjoint partition of $\mathbb{R}^{n}$ (see ${ }^{7}$ and ${ }^{9}$ for more details).

We say that $g_{Q}$ is a generalized Haar function associated with $Q \in \mathscr{D}$ if

(a) $\operatorname{supp}\left(g_{Q}\right) \subset Q$;

(b) if $Q^{\prime} \in \mathscr{D}$ and $Q^{\prime} \subsetneq Q$, then $g_{Q}$ is constant on $Q^{\prime}$;

(c) $\left\|g_{Q}\right\|_{\infty} \leq 1$.

Given a dyadic grid $\mathscr{D}$ and a pair $(m, k) \in \mathbb{Z}_{+}^{2}$, a linear operator $\mathcal{S}$ is a generalized Haar shift operator (that is, a dyadic Calderón-Zygmund operator) of complexity type $(m, k)$ if it is bounded on $L^{2}\left(\mathbb{R}^{n}\right)$ and

$$
\mathcal{S} f(x)=\sum_{Q \in \mathscr{D}} \mathcal{S}_{Q} f(x)=\sum_{\substack{Q \in \mathscr{D} \\ Q^{\prime} \in \mathscr{D}_{m}(Q) \\ Q^{\prime \prime} \in \mathscr{D}_{k}(Q)}} \frac{\left\langle f, g_{Q^{\prime}}^{Q^{\prime \prime}}\right\rangle}{|Q|} g_{Q^{\prime \prime}}^{Q^{\prime}}(x),
$$


where $\mathscr{D}_{j}(Q)$ stands for the dyadic subcubes of $Q$ with side length $2^{-j} \ell(Q)$, $g_{Q^{\prime}}^{Q^{\prime \prime}}$ is a generalized a Haar function associated with $Q^{\prime}$ and $g_{Q^{\prime \prime}}^{Q^{\prime}}$ is a generalized a Haar function associated with $Q^{\prime \prime}$. We say that the complexity of $\mathcal{S}$ is $\kappa=\max (m, k)$. We also define the truncated Haar shift operator

$$
\mathcal{S}_{\star} f(x)=\sup _{0<\epsilon<\epsilon^{\prime}<\infty}\left|\mathcal{S}_{\epsilon, \epsilon^{\prime}} f(x)\right|=\sup _{0<\epsilon<\epsilon^{\prime}<\infty}\left|\sum_{\substack{Q \in \mathscr{D} \\ \epsilon \leq \ell(Q) \leq \epsilon^{\prime}}} \mathcal{S}_{Q} f(x)\right| .
$$

An important example of a Haar shift operator on the real line is the Haar shift (also known as the dyadic Hilbert transform) $H^{d}$, defined by

$$
H^{d} f(x)=\sum_{I \in \Delta}\left\langle f, h_{I}\right\rangle\left(h_{I_{-}}(x)-h_{I_{+}}(x)\right),
$$

where, given a dyadic interval $I, I_{+}$and $I_{-}$are its right and left halves, and

$$
h_{I}(x)=|I|^{-1 / 2}\left(\chi_{I_{-}}(x)-\chi_{I_{+}}(x)\right) .
$$

After renormalizing, $h_{I}$ is a Haar function on $I$ and one can write $H^{d}$ as a generalized Haar shift operator of complexity 1 . These operators have played a very important role in the proof of the $A_{2}$ conjecture: see $6,10,11$ and the references they contain for more information.

Associated with the dyadic grid $\mathscr{D}$ is the dyadic maximal function

$$
M_{\mathscr{D}} f(x)=\sup _{x \in Q \in \mathscr{D}} f_{Q}|f(y)| d y .
$$

Note that $M_{\mathscr{D}}$ is dominated pointwise by the Hardy-Littlewood maximal operator.

We can now state our result for dyadic Calderón-Zygmund operators.

Theorem 1.2. Let $\mathcal{S}$ be a generalized Haar shift operator of complexity $\kappa$. Given $1<p<q<\infty$ and a pair of weights $(u, v)$, if the dyadic maximal operator satisfies

$$
M_{\mathscr{D}}: L^{p}(v) \rightarrow L^{q}(u) \quad \text { and } \quad M_{\mathscr{D}}: L^{q^{\prime}}\left(u^{1-q^{\prime}}\right) \rightarrow L^{p^{\prime}}\left(v^{1-p^{\prime}}\right),
$$

then

$$
\|\mathcal{S} f\|_{L^{q}(u)} \leq C \kappa^{2}\|f\|_{L^{p}(v)} \quad \text { and } \quad\left\|\mathcal{S}_{\star} f\right\|_{L^{q}(u)} \leq C \kappa^{2}\|f\|_{L^{p}(v)} .
$$

Analogously, if the dyadic maximal operator satisfies

$$
M_{\mathscr{D}}: L^{q^{\prime}}\left(u^{1-q^{\prime}}\right) \rightarrow L^{p^{\prime}}\left(v^{1-p^{\prime}}\right)
$$

then

$$
\|\mathcal{S} f\|_{L^{q, \infty}(u)} \leq C \kappa^{2}\|f\|_{L^{p}(v)} \quad \text { and } \quad\left\|S_{\star} f\right\|_{L^{q, \infty}(u)} \leq C \kappa^{2}\|f\|_{L^{p}(v)} .
$$




\section{Proofs of the Main results}

\section{Proof of Theorem 1.1}

We will prove our estimates for $T_{\star}$; the ones for $T$ are completely analogous.

Given a dyadic grid $\mathscr{D}$ we say that $\left\{Q_{j}^{k}\right\}_{j, k}$ is a sparse family of dyadic cubes if for any $k$ the cubes $\left\{Q_{j}^{k}\right\}_{j}$ are pairwise disjoint; if $\Omega_{k}:=\cup_{j} Q_{j}^{k}$, then $\Omega_{k+1} \subset \Omega_{k}$; and $\left|\Omega_{k+1} \cap Q_{j, k}\right| \leq \frac{1}{2}\left|Q_{j}^{k}\right|$. Given $\mathscr{D}$ and a sparse family $\mathscr{S}=\left\{Q_{j}^{k}\right\}_{j, k} \subset \mathscr{D}$, define the positive dyadic operator $\mathscr{A}$ by

$$
\mathscr{A} f(x)=\mathscr{A}_{\mathscr{D}, \mathscr{S}} f(x)=\sum_{j, k} f_{Q_{j}^{k}} \chi_{Q_{j}^{k}}(x)
$$

where $f_{Q}=f_{Q} f(y) d y$.

For our proof we will use the main result in ${ }^{7,9}$. Given a Banach function space $X$ and a non-negative function $f$,

$$
\left\|T_{\star} f\right\|_{X} \leq C(T, n) \sup _{\mathscr{D}, \mathscr{S}}\left\|\mathscr{A}_{\mathscr{D}, \mathscr{S}} f\right\|_{X}
$$

where the supremum is taken over all dyadic grids $\mathscr{D}$ and sparse families $\mathscr{S} \subset \mathscr{D}$. To prove Theorem 1.1 we apply this result with $X=L^{q}(u)$ or $X=L^{q, \infty}(u)$; it will then suffice to show that our assumptions on $M$ guarantee that $\mathscr{A}_{\mathscr{D}, \mathscr{S}}$ satisfies the corresponding two weight inequalities.

To prove this fact we will use a result by Lacey, Sawyer and UriateTuero $^{12}$. Given a sequence of non-negative constants $\alpha=\left\{\alpha_{Q}\right\}_{Q \in \mathscr{D}}$, define the positive operator

$$
T_{\alpha} f(x)=\sum_{Q \in \mathscr{D}} \alpha_{Q} f_{Q} \chi_{Q}(x)
$$

Further, given $R \in \mathscr{D}$ we define the "outer truncated" operator

$$
T_{\alpha}^{R} f(x)=\sum_{\substack{Q \in \mathscr{D} \\ \text { mathcal } \supset R}} \alpha_{Q} f_{Q} \chi_{Q}(x)
$$

In ${ }^{12}$ it was shown that for all $1<p<q<\infty, T_{\alpha}: L^{p}(v) \rightarrow L^{q}(u)$ if and only if there exist constants $C_{1}$ and $C_{2}$ such that for every $R \in \mathscr{D}$

$$
\left(\int_{\mathbb{R}^{n}} T_{\alpha}^{R}\left(v^{1-p^{\prime}} \chi_{R}\right)(x)^{q} u(x) d x\right)^{\frac{1}{q}} \leq C_{1}\left(\int_{R} v(x)^{1-p^{\prime}} d x\right)^{\frac{1}{p}}
$$

and

$$
\left(\int_{\mathbb{R}^{n}} T_{\alpha}^{R}\left(u \chi_{R}\right)(x)^{p^{\prime}} v(x)^{1-p^{\prime}} d x\right)^{\frac{1}{p^{\prime}}} \leq C_{2}\left(\int_{R} u(x) d x\right)^{\frac{1}{q^{\prime}}}
$$


Furthermore, for $1<p<q<\infty, T_{\alpha}: L^{p}(v) \rightarrow L^{q, \infty}(u)$ holds if and only if there exists a constant $C_{2}$ such that for every $R \in \mathscr{D}$, (11) holds.

We can apply these results to the operator $\mathscr{A}=\mathscr{A} \mathscr{D}, \mathscr{S}$ where $\mathscr{D}$ and $\mathscr{S}$ are fixed, since $\mathscr{A}=T_{\alpha}$ with $\alpha_{Q}=1$ if $Q \in \mathscr{S}$ and $\alpha_{Q}=0$ otherwise. Fix $R \in \mathscr{D}$; to estimate $\mathscr{A}^{R}$, take the increasing family of cubes $R=R_{0} \subsetneq R_{1} \subsetneq R_{2} \subsetneq \ldots$ with $R_{k} \in \mathscr{D}$ and $\ell\left(R_{k}\right)=2^{k} \ell(R)$. Define $R_{-1}=\varnothing$. Note that $\operatorname{supp} \mathscr{A}^{R} \subset \cup_{k \geq 0} R_{k}$. Then for every non-negative function $f$ and for every $x \in R_{k} \backslash R_{k-1}$ with $k \geq 0$ we have that

$$
\begin{aligned}
0 \leq \mathscr{A}^{R}\left(f \chi_{R}\right)(x) \leq \sum_{j=0}^{\infty}\left(f \chi_{R}\right)_{R_{j}} \chi_{R_{j}}(x)= & f_{R} \sum_{j=k}^{\infty} 2^{-j n} \\
& \lesssim f_{R} 2^{-k n}=\left(f \chi_{R}\right)_{R_{k}} \leq M_{\mathscr{D}}\left(f \chi_{R}\right)(x) .
\end{aligned}
$$

Consequently, for every $x \in \mathbb{R}^{n}$,

$$
0 \leq \mathscr{A}^{R}\left(f \chi_{R}\right)(x) \lesssim M_{\mathscr{D}}\left(f \chi_{R}\right)(x) \leq M\left(f \chi_{R}\right)(x) .
$$

Inequality (12) together with our hypothesis (1) implies (10) and (11). Therefore, we have that $\mathscr{A}: L^{p}(v) \rightarrow L^{q}(u)$ with constants depending on the dimension, $p, q$ and the implicit constants in (1). Therefore, by Lerner's estimate (9) we get $T_{\star}: L^{p}(v) \rightarrow L^{q}(u)$ as desired.

For the weak-type estimates we proceed in the same manner, using the fact that (3) yields (11) and therefore $\mathscr{A}: L^{p}(v) \rightarrow L^{q, \infty}(u)$. This in turn implies, by Lerner's estimate (9) applied to $X=L^{q, \infty}(u)$, that $T_{\star}: L^{p}(v) \rightarrow L^{q, \infty}(u)$.

\section{Proof of Theorem 1.2}

Fix $\mathscr{D}$ and a generalized Haar shift operator of complexity $\kappa$. As before we can work with $\mathcal{S}_{\star}$. We can repeat the previous argument except that we want to keep the fixed dyadic structure $\mathscr{D}$. A careful examination of ${ }^{7}$ Section 5 shows that, given a Banach function space $X$, we have

$$
\left\|\mathcal{S}_{\star} f\right\|_{X} \leq C_{n} \kappa^{2} \sup _{\mathscr{S}}\left\|\mathscr{A}_{\mathscr{D}, \mathscr{S}} f\right\|_{X}, \quad f \geq 0
$$

where the supremum is taken over all sparse families $\mathscr{S} \subset \mathscr{D}$. We emphasize that in ${ }^{7}$ Section 5 there is an additional supremum over the dyadic grids $\mathscr{D}$. This is because at some places the dyadic maximal operator is majorized by the regular Hardy-Littlewood maximal operator and the latter is in turn controlled by a sum of $\mathscr{A}_{\mathscr{D}_{\alpha}, \mathscr{S}_{\alpha}}$ for $2^{n}$ dyadic grids $\mathscr{D}_{\alpha}$. However, keeping $M_{\mathscr{D}}$ one can easily show that (13) holds. Details are left to the interested reader. 
Given (13), we fix a sparse family $\mathscr{S} \subset \mathscr{D}$ and write $\mathscr{A}=\mathscr{A} \mathscr{D}, \mathscr{S}$. Arguing exactly as before we obtain (12). Thus, (5) implies (10) and (11) and therefore the result from ${ }^{12}$ yields $\mathscr{A}: L^{p}(v) \rightarrow L^{q}(u)$ with constants depending on the dimension, $p, q$ and the implicit constants in (5). Combining this with Lerner's estimate (13) applied to $X=L^{q}(u)$ we conclude as desired that $\mathcal{S}_{\star}: L^{p}(v) \rightarrow L^{q}(u)$. We get the weak-type estimate by adapting the above proof in exactly the same way.

\section{References}

1. M. C. Reguera and J. Scurry, On joint estimates for maximal functions and singular integrals on weighted spaces, Proc. Amer. Math. Soc. 141, 1705 (2013).

2. D. Cruz-Uribe, A. Reznikov and A. Volberg, Logarithmic bump conditions and the two-weight boundedness of Calderón-Zygmund operators, Adv. Math. 255, 706 (2014).

3. D. Cruz-Uribe, J. M. Martell and C. Pérez, Sharp two-weight inequalities for singular integrals, with applications to the Hilbert transform and the Sarason conjecture, Adv. Math. 216, 647 (2007).

4. D. Cruz-Uribe, J. M. Martell and C. Pérez, Sharp weighted estimates for approximating dyadic operators, Electron. Res. Announc. Math. Sci. 17, 12 (2010).

5. D. Cruz-Uribe, J. Martell and C. Pérez, Weights, extrapolation and the theory of Rubio de Francia, Operator Theory: Advances and Applications, Vol. 215 (Birkhäuser/Springer Basel AG, Basel, 2011).

6. D. Cruz-Uribe, J. Martell and C. Pérez, Sharp weighted estimates for classical operators, Adv. in Math 229, 408 (2012).

7. A. K. Lerner, On an estimate of Calderón-Zygmund operators by dyadic positive operators, J. Anal. Math. 121, 141 (2013).

8. E. Sawyer, A characterization of a two-weight norm inequality for maximal operators, Studia Math. 75, 1 (1982).

9. A. K. Lerner, A simple proof of the $A_{2}$ conjecture, Int. Math. Res. Not. IMRN 14, 3159 (2013).

10. T. P. Hytönen, The sharp weighted bound for general CalderónZygmund operators, Ann. of Math. (2) 175, 1473 (2012).

11. T. Hytönen, C. Pérez, S. Treil and A. Volberg, Sharp weighted estimates for dyadic shifts and the $A_{2}$ conjecture, J. Reine Angew. Math. 687, 43 (2014).

12. M. T. Lacey, E. T. Sawyer, C.-Y. Shen and I. Uriarte-Tuero, 
Two-weight inequality for the Hilbert transform: a real variable characterization, I, Duke Math. J. 163, 2795 (2014). 